\title{
Essais
}

Revue interdisciplinaire d'Humanités

Hors-série 1 | 2013

L'estrangement

\section{Familiarité et estrangement : de faux antonymes}

Hélène Merlin-Kajman

\section{OpenEdition}

Journals

Édition électronique

URL : http://journals.openedition.org/essais/2422

DOI : 10.4000/essais.2422

ISSN : 2276-0970

\section{Éditeur}

École doctorale Montaigne Humanités

\section{Édition imprimée}

Date de publication : 15 octobre 2013

Pagination : 161-179

ISBN : 978-2-9544269-7-4

ISSN : 2417-4211

Référence électronique

Hélène Merlin-Kajman, «Familiarité et estrangement : de faux antonymes », Essais [En ligne], Horssérie 1 | 2013, mis en ligne le 05 octobre 2020, consulté le 12 octobre 2020. URL : http:// journals.openedition.org/essais/2422 ; DOI : https://doi.org/10.4000/essais.2422 


\section{Familiarité et estrangement : de faux antonymes}

\section{Hélène Merlin-Kajman}

Dans le chapitre "L'estrangement » de son recueil de textes paru en français sous le titre $\grave{A}$ distance $^{1}$, Carlo Ginzburg fait la généalogie de ce concept par lequel, en $1925^{2}$, le critique russe Viktor Chklovski a défini un processus artistique majeur consistant à renouveler la perception du monde en le regardant et le montrant d'un point de vue inhabituel. "La voie passablement tortueuse que j'emprunterai... » : c'est par cette expression que Carlo Ginzburg amorce son trajet, qui vise à réinscrire l'analyse formelle et a-chronique de Chklovski dans une continuité historique, le plaçant lui-même de la sorte, quoique de façon encore implicite, sous le signe de l'estrangement. Je ne sais pas si l'on peut bifurquer dans des voies tortueuses, mais pour filer la métaphore du fil qu'affectionne tant l'historien, je vais séparer des fils tissés ensemble dans cet article et $\mathrm{y}$ introduire deux bifurcations qui me mèneront à quelques questions, à partir desquelles je ferai retour à la démarche de Carlo Ginzburg pour insister finalement sur un aspect de sa démarche qui se place non pas sous le signe de la distance, nécessaire au travail de l'historien selon lui, mais au contraire sous le signe du rapprochement, de la vision rapprochée, voire de la familiarité. S'il s'agit là d'un geste apparemment inverse à celui de la prise de distance, j'aimerais suggérer qu'il n'est peut-être pas inverse à celui de l'estrangement, et ceci en deux sens presque opposés que nos bifurcations nous aurons permis d'apercevoir clairement, du moins je l'espère.

1 Carlo Ginzburg, À Distance. Neuf essais sur le point de vue en histoire, trad. Pierre-Antoine Fabre, Paris, Gallimard, 2001.

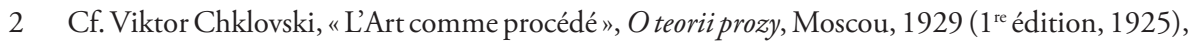
in Théorie de la littérature. Textes des formalistes russes, présentés et traduits par Tzvetan Todorov (préface de Roman Jakobson), Paris, Seuil, «Tel quel », 1965. Tzvetan Todorov a traduit ostranienie, traduit ici par " estrangement », par «singularisation " (cf. index p. 315, "Procédé de singularisation "; et note du traducteur Pierre-Antoine Fabre in Carlo Ginzburg, À Distance, op. cit., p. 15). On rencontre aussi «étrangisation » et « défamiliarisation». 
Pour Chklovski, le procédé d'estrangement consiste à sortir nos perceptions de leur automatisme habituel, à décrire un objet, une personne ou une situation comme s'ils étaient vus pour la première fois ou d'un point de vue inédit (par un étranger notamment) voire impossible (par un animal par exemple) afin d'empêcher que fonctionne, à leur égard, le processus réflexe de reconnaissance : le procédé les ramène, dans toute leur consistance étonnante, à la pleine conscience du lecteur. Il s'agit là de produire, résume Carlo Ginzburg, "une tension cognitive qui révèle peu à peu les traits imprévisiblement étranges d'un objet familier ${ }^{3}$ ".

La voie tortueuse de l'historien nous conduit des exemples donnés par Chklovski (empruntés à Tolstoï pour l'essentiel) aux pensées de Marc Aurèle, et de là, au faux livre de Marc Aurèle écrit par Guevara et publié en Espagne en 1520, plus particulièrement à la harangue supposément prononcée devant l'empereur et le sénat par un paysan du Danube venu protester contre l'impérialisme romain. À propos de cette dernière, Carlo Ginzburg souligne que, quoique la harangue du paysan du Danube «ne sui[ve] pas une première phase d'opacité et d'incompréhension ${ }^{4}$ " contrairement aux exemples donnés par Chklovski, de nombreux cas d'estrangement littéraire postérieurs à Guevara tirent de son modèle une particularité : "Depuis ce moment, le sauvage, le paysan et l'animal, ensemble ou séparément, ont constitué un point de vue pour l'observation de la société d'un œil distant, étonné et critique $»$. Un paragraphe plus haut, Carlo Ginzburg avait également évoqué « la tradition populaire médiévale " où " le roi est défié par un paysan dont l'aspect grotesque est imprévisiblement marié à la finesse et à la sagesse "; et il avait cité plus particulièrement les Sottilissime astuzie di Bertoldo de Giulio Cesare Croce où Bertoldo fait au roi une répartie ridiculisante qui compare les seigneurs et barons qui l'entourent à des fourmis entourant le sorbier et en rongeant l'écorce :

Ces comparaisons animalières visent à rabaisser l'autorité du roi, thème analysé en profondeur par Bakhtine dans son grand livre sur la culture populaire à la Renaissance ${ }^{6}$.

Carlo Ginzburg ne s'étend pas davantage sur Bakhtine. Pourtant, il me paraît manifeste que le concept de carnavalesque dégagé par ce dernier à partir de l'œuvre de Rabelais recoupe à maints égards celui d'estrangement. Pour Bakhtine, le rire carnavalesque empêche le figement de l'existence quotidienne dans le sérieux de la vie officielle et de ses formes stéréotypées : il « jet[te] un

3 Carlo Ginzburg, op. cit., p. 28.

4 Ibid., p. 25.

5 Ibid., p. 26. Un autre article de Chklovski, "La construction de la nouvelle et du roman ", mentionne cependant que "l'ancien roman grec connaissait lui aussi ce procédé lorsqu'il décrivait la ville du point de vue d'un paysan " (Théorie de la littérature, Textes des formalistes russes, op. cit., p. 186).

6 Carlo Ginzburg, op. cit., p. 25. 
regard neuf sur [elles] $]^{7}$ ", il les " purifie du dogmatisme, du caractère unilatéral, de la sclérose, du fanatisme et de l'esprit catégorique, des éléments de peur ou d'intimidation, du didactisme, de la naïveté et des illusions, d'une néfaste fixation sur un plan unique, de l'épuisement stupide ${ }^{8}$ ". Il s'agit bien de changer de point de vue pour faire surgir une vision de la réalité renouvelée, ici, par le rire. Le carnavalesque bouleverse l'ordre automatique des représentations, les explications du monde stabilisées qui fondent la société sur une hiérarchie divine pesant de tout son poids terrifiant sur les hommes. Et l'on peut dire de lui ce que Chklovski dit de l'estrangement : qu'il « ressuscit[e] notre perception de la vie " et rend "les choses à nouveau sensibles ${ }^{9}$ ". Une phrase de Chklovski : "L'art est le moyen de voir quelque chose devenir ; ce qui a réellement été n'a aucune importance ${ }^{10}$ " rencontre encore plus d'écho avec la réflexion de Bakhtine, pour qui le rire carnavalesque est par excellence un opérateur de devenir, de mouvement, de changement : ou plutôt, l'opérateur par lequel se révèle que la vie est en perpétuel devenir et mouvement, se confond même avec ce mouvement, de la naissance à la mort, en passant par tous les états possibles et imaginables du corps grotesque, non ce décor de formes stables, stabilisées, dont la vie officielle essaie d'imposer la régularité morte. Du reste, Carlo Ginzburg rappelle l'importance de la devinette à sous-entendu érotique dans les exemples analysés par Chklovski : ceci nous rapproche encore du carnavalesque selon Bakhtine.

Dans le cheminement de Carlo Ginzburg, le détour initial par l'empereur stoïcien Marc Aurèle, avait, à mon sens, préparé ce bref rapprochement entre l'estrangement et le carnavalesque. Marc Aurèle, nous dit en effet Carlo Ginzburg, propose une technique morale qui passe par une démarche de défamiliarisation d'avec les représentations habituelles, défamiliarisation résumée dans la formule suivante : "Souviens-toi de pénétrer toute chose jusqu'à chacune de ses parties et, par cette analyse, de parvenir à la mépriser ; et applique la même opération à la vie dans son ensemble ${ }^{11}$ ". La défamiliarisation passe donc par la dégradation imaginaire de la totalité et de sa belle forme, comme l'indique clairement un autre passage cité par Carlo Ginzburg:

7 Mikhail Bakhtine, L'œuvre de François Rabelais et la culture populaire au Moyen Âge et sous la Renaissance, Paris, Gallimard, 1973, p. 434-435.

8 Ibid., p. 127.

9 Cité par Carlo Ginzburg, op. cit., p. 16.

10 Ibid., loc. cit. "Et voilà que pour rendre la sensation de la vie, pour sentir les objets, pour éprouver que la pierre est de pierre, il existe ce que l'on appelle l'art. Le but de l'art, c'est de donner une sensation de l'objet comme vision et non pas comme reconnaissance ; le procédé de l'art est le procédé de singularisation des objets et le procédé qui consiste à obscurcir la forme, à augmenter la difficulté et la durée de la perception. L'acte de perception en art est une fin en soi et doit être prolongé ; l'art est un moyen d'éprouver le devenir de l'objet, ce qui est déjà "devenu” n'importe pas pour l'art. " (Viktor Chklovski, «L'Art comme procédé », art. cit., p. 83)

11 Carlo Ginzburg, op. cit., p. 19. 
De même que l'on peut se représenter [phantasein] une friandise ou tout autre mets en se disant : " ceci est le cadavre d'un poisson, ou d'un oiseau, ou d'un cochon ", [...] ou bien : "ce laticlave est du poil de brebis trempé dans le jus d'un coquillage "; ou se dire de l'accouplement : " c'est le frottement d'un boyau et une sécrétion de liquide gluant accompagnée d'un spasme ». De même que ces représentations touchent aux choses-mêmes, les pénètrent jusqu'au fond et en dévoilent la vraie nature, de même il faut, toute la vie durant, quand les choses qui se présentent à nous se font trop persuasives, les mettre à nu, voir le peu qu' elles sont et les dépouiller de la fiction par laquelle elles se rendent si vénérables (VI, 13) ${ }^{12}$.

Pour commenter des citations de Voltaire, de La Bruyère ${ }^{13}$, et plus loin de Tolstoï qui procèdent, selon Carlo Ginzburg, d'un même mouvement de "délégitimation à tous les niveaux, politique, social, religieux ${ }^{14}$ " que les pensées de Marc Aurèle, l'historien reprend à son compte l'idée de l'empereur stoïcien selon laquelle cet estrangement permet d'atteindre les " "choses elles-mêmes et de les pénétrer jusqu'au fond et dévoiler leur vraie nature", jusqu’à "les mettre à nu, voir le peu qu'elles sont et les dépouiller de la fiction par laquelle elles se rendent si vénérables" 15 ». Et il ajoute que l'enjeu, c'est, " en dernière analyse, l'acceptation de la finitude et de la mort ${ }^{16}{ }^{\prime}$. Même si le rire n'est pas l'horizon de Marc Aurèle ni de Voltaire, La Bruyère ou Tolstoï dans les exemples envisagés par Carlo Ginzburg (contrairement aux devinettes à sous-entendu érotique), cette remarque confirme la pertinence du rapprochement du carnavalesque et de l'estrangement puisque le carnavalesque révèle la finitude humaine et le corps grotesque derrière l'habit des dignités. Même si le carnavalesque ajoute de la joie là où le stoïcisme programme du mépris, et une forme d'adhésion à la vie là où le second ne vise qu'au détachement à son égard, ce à quoi l'un et l'autre barrent la route, c'est aux alibis esthétiques, moraux, sociaux, qui embellissent (tel est le point de vue induit) des apparences auxquelles ils sont au contraire chargés de rendre leur caractère bas ou trivial. Et la page de Résurrection de Tolstoï sur laquelle s'attarde un moment Carlo Ginzburg me parait constituer un remarquable trait d'union entre les deux catégories :

[...] le prêtre, affublé d'un costume de brocart spécial, étrange et très incommode, découpait du pain en petits morceaux qu'il disposait dans une soucoupe, pour les tremper ensuite dans une coupe de vin, tout en prononçant des noms divins et des prières ${ }^{17}$.

12 Ibid., p. 20.

13 Il s'agit du célèbre fragment 128 du chapitre « De l'Homme » des Caractères, qui commence par "L'on voit certains animaux farouches, des mâles et des femelles, répandus par la campagne, noirs, livides [...] ils montrent une face humaine, et en effet ils sont des hommes. " Jean de La Bruyère, Les Caractères, Paris, GF Flammarion, 1965, p. 292.

14 Carlo Ginzburg, op. cit., p. 29.

15 Ibid., p. 30.

16 Ibid., loc. cit.

17 Cité par Carlo Ginzburg, ibid., loc. cit. 
Ce passage décrit le rite religieux non selon le point de vue cérémoniel et sacré des fidèles mais selon un point de vue naïf, estrangé, qui prend ce qui est vu à la lettre : et la profanation littéraire s'appuie sur une sorte de carnavalisation grotesque du " corps » mystique de l'eucharistie.

Mais c'est ici que je vais amorcer ma première bifurcation. En effet, dans les exemples de Marc Aurèle comme dans celui de Tolstoï, je suis moins frappée par le gain cognitif de l'estrangement que par l'opération de dégradation burlesque auxquels se livrent leurs auteurs. Le meilleur des estrangements, la meilleure des mises à distance, passeraient-ils par la ridiculisation, le rabaissement de ce qui est haut ? On peut en tout cas se demander si l'estrangement est possible en dehors d'un monde où des différences, la différence du haut et du bas par exemple, ou encore la différence entre un plan de dignité immortelle et un plan de simple mortalité, existent préalablement à lui. Dans cette hypothèse, l'estrangement consisterait d'abord à ramener toutes ces différences à un même niveau, à les égaliser, à les annuler par le bas, à faire chuter catégorisations et symboles dans l'identité d'un concret commun, du charnel, du mortel, bref, de ce que Bakhtine appelle le corps grotesque. En fait, la suite de la réflexion de Carlo Ginzburg nous emmène dans une toute autre direction dans laquelle je ne vais pas tarder à m'engager aussi. Mais auparavant, continuons à suivre un peu mon premier chemin de traverse.

Car voici la source de mon étonnement : Bakhtine n'appelle pas cette culture une culture de l'estrangement. Il l'associe paradoxalement à la familiarité. Contrairement à que l'on retient souvent de son livre à cause de l'importance qu'y revêt l'exemple du carnaval pour dégager ses traits spécifiques, cette culture du rire n'apparaît pas le moins du monde réservée à quelques moments festifs : selon lui, elle est au contraire quotidienne, familière, populaire, partagée - comme la description du service divin par Tolstoï, on peut le remarquer : car le décrire selon un plan étranger à sa sacralité théorique, c'est le ramener au quotidien, au familier du pain concret et des gestes littéraux.

Face à la culture officielle, le carnavalesque est donc la familiarité même, et c'est quand on retrouve cette familiarité que l'on peut, comme dans un réveil ou dans un sursaut bienfaisants, trouver si étrange et burlesque la culture officielle. Culture populaire, culture familière qui fait du peuple, ou le montre comme, une seule famille, cette culture est même le nom d'un certain type de partage, le seul partage valide aux yeux de Bakhtine : le partage fusionnel du peuple entier dans des gestes, actions, langage, qui rappellent joyeusement la mortalité de la créature humaine, c'est-à-dire aussi sa perpétuelle renaissance.

Le modèle de Bakhtine est heureux et postule une fondamentale innocence du peuple opprimé. Mais il s'agit là d'une utopie qui cache une face sombre ${ }^{18}$.

18 Cf. mes articles «Peur, rire et outrage : la face sombre de la "culture carnavalesque" », Textuel, « Peur et littérature du Moyen Âge au XVII siècle ", Pascal Debailly et Florence Dumora (éd.), n 51, juin 2007 ; " Oublier le carnavalesque », in " Retour à Bakhtine. Essais de lectures bakhtiniennes ", Textuel, n 69. Textes réunis par Marc Hersant et Chantal Liaroutzos, 2012. 
Dans Les guerriers de Dieu, l'historien Denis Crouzet a analysé l'étroite connexion existant, pendant les guerres de religion, entre des rites d'inversion, le rire carnavalesque, et des actes d'extrême violence physique commise surtout par les catholiques sur les protestants. L'intervention fréquente des enfants dans les massacres constitue en effet le point de départ de sa réflexion portant sur des violences jusqu'alors expliquées par des causes sociales, économiques ou politiques. Il montre comment le caractère profondément étrange, inhabituel, du rôle " justicier " des enfants dans les massacres a appelé, de la part des catholiques, une interprétation non moins étrange. Ils y ont lu un signe de la présence sacrale de Dieu, un Dieu se révélant, en somme, comme puissance carnavalesque de réveil et comme opérateur d'anomie. Les violences des guerres civiles ne s'effectuent que sur fond de cette double dimension de familiarisation dégradante et de défamiliarisation :

\begin{abstract}
[...] en 1562, le 4 juillet, des catholiques s'emparent de la chaire du ministre Sorel et se mettent à parodier une exécution capitale, malgré l'absence de Sorel. Le ministre se voit assimilé à la figure de Carême, ce parce que "voulant faire preuve d'esprit et de bon goût, ils suspendirent à cette chaire un hareng saur, dont le nom dérivait du pasteur Sorel ». Le hareng saur, tout comme la morue, est une personnification de Carême. [...] [L]e glissement phonétique de Sorel à saur devait être d'autant plus comique qu'il jouait doublement sur les mots, et que le rire s'amplifiait de la désacralisation même de la religion ennemie : c'est une sorte de carnaval absurde qui est mise en scène, parce qu'inversée en sa propre symbolique. Carême-Sorel ne combat pas la chaire, il est assis sur elle, sur la chaire-chair, promenée alors triomphalement. L'homme de la chaire est homme de chair ${ }^{19}$.
\end{abstract}

Ici, le ministre protestant, et son ministère, deviennent étrangers à la communauté non seulement chrétienne, mais encore humaine, et cet estrangement réel, réellement effectué, est motivé par une sorte de devinette en acte, un jeu de mots familier, ridiculisant et rabaissant, le calembour sur le nom propre de Sorel.

L'erreur de perspective de Bakhtine dans sa lecture utopique du carnavalesque vient de ce qu'il sous-estime la violence toujours latente de la familiarité parce qu'il ne voit pas qu'elle est structurellement liée à la culture officielle : elle ne la transgresse pas, elle ne la subvertit pas, parce qu'il faut plutôt dire que la culture est constituée par l'entrelacement et la tension entre ces deux registres : fusion rieuse et distance respectueuse.

On touche ici à un phénomène bien connu des anthropologues. Dès 1928, Marcel Mauss, dans un article intitulé "Parentés à plaisanteries ${ }^{20}$ ", a mis en évidence l'existence, dans les sociétés dites primitives, de deux types

19 Denis Crouzet, Les guerriers de Dieu. La violence au temps des troubles de religion. Vers 1525-vers 1610, t. 1, Paris, Champ Vallon, 1990, p. 341-342.

20 Marcel Mauss, «Parentés à plaisanteries », in Essais de sociologie, Paris, Minuit, « Essais/Points », 1968 et 1969. 
de relation inverses et complémentaires : alors que certaines parentés, par exemple la relation de belle-mère à gendre, sont marquées par des formes de déférence et de respect, d'autres parentés, par exemple les relations entre beaux-frères et belles-sœurs, sont au contraire marquées par la familiarité et la plaisanterie. Ces styles de relation ne sont pas laissés au libre choix des individus, mais dépendent de règles sociales organisant à la fois non seulement les relations " à respect " mais aussi les relations "à plaisanteries " malgré leur apparente spontanéité : l'ensemble des relations au sein du groupe se trouvent donc distribuées selon une grammaire antithétique qui articule les individus de façon sélective et réciproque.

Il s'agit là d'un partage du sensible - pour reprendre ce syntagme à Rancière ${ }^{21}$ en le déplaçant - en quelque sorte pré-politique, sociétal. Car pour Mauss, ces échanges de gestes et de mots au cours desquels se donnent et se rendent respects et politesses ou bien plaisanteries et injures doivent être compris comme les éléments les plus primitifs du système du don auquel il a consacré un essai célèbre $^{22}$. Les premiers " dons " de l'histoire de l'humanité seraient donc des dons de paroles et ils auraient une finalité tout à fait spécifique : celle d'établir et de manifester des liens sociaux différents mais complémentaires. Le phénomène, conclut Mauss, laisse " entrevoir une façon d'étudier certaines des mœurs les plus générales ": non seulement bien sûr les relations interindividuelles (étiquette $v s$ familiarité, respect $v s$ ridicule, etc.) dans toutes sortes de sociétés, mais encore « la nature et la fonction d'éléments esthétiques importants, mêlés naturellement, comme partout, aux éléments moraux de la vie sociale » :

Les obscénités, les chants satiriques, les insultes envers les hommes, les représentations ridicules de certains êtres sacrés sont d'ailleurs à l'origine de la comédie ; tout comme les respects témoignés aux hommes, aux dieux et aux héros nourrissent le lyrique, l'épique, le tragique ${ }^{23}$.

La réflexion de Marcel Mauss ouvre à mon sens une perspective décisive pour la compréhension de l'estrangement " littéraire » (ou du moins de certains de ses aspects les plus puissants) en le replaçant dans des expériences sociales ordinaires et polarisées, nous révélant ainsi que l'ordinaire lui-même repose sur une discordance, la discordance entre deux styles de relations antithétiques, dont l'une a, de plus, le privilège bizarre de rapprocher et de rendre familier ce qui était lointain (et respectable), voire de rabaisser ce qui était haut par un procédé de mélange et de contamination des deux styles, ce qui annule le pôle " haut ». Rabaisser ce qui était haut, c'est en faire ressortir l'étrangeté, mais par le procédé d'une familiarisation inhabituelle - qui est cependant typique

21 Cf. Jacques Rancière, Le partage du sensible. Esthétique et politique, Paris, La Fabrique, 2000.

22 Cf. Marcel Mauss, Essai sur le don, in Sociologie et anthropologie, Paris, PUF, 2006 (1 ${ }^{\text {re }}$ édition, 1950), p. 227.

23 Marcel Mauss, «Parentés à plaisanteries », op. cit., p. 161. 
de certains jeux familiers $^{24}$. Car, il faut le souligner, le régime de la familiarité repose fréquemment sur le jeu avec des discordances. Voici un exemple simple de cette dynamique - et de ses enjeux - emprunté au XVII ${ }^{e}$ siècle, un siècle où certaines relations peuvent être soit à plaisanterie, soit à respect, en fonction des situations et non pas uniquement des statuts des personnes considérées, phénomène de chevauchement, par rapport à la situation simple décrite par Mauss, rendu possible par le christianisme qui a dédoublé l'être humain lui-même entre deux pôles ${ }^{25}$.

Dans une lettre que $\mathrm{M}^{\mathrm{me}}$ de Sévigné adresse à sa fille, $\mathrm{M}^{\mathrm{me}}$ de Grignan, alors que cette dernière a rejoint son mari, lieutenant général de la Provence, et que son beau-frère, le coadjuteur d'Arles, avec qui elle est en froid, séjourne auprès du couple, $\mathrm{M}^{\mathrm{me}}$ de Sévigné s'inquiète :

Et notre coadjuteur, ne voulez-vous pas bien l'embrasser pour l'amour de moi ? N'est-il point encore Seigneur Corbeau pour vous ? Je désire avec passion que vous soyez remis comme vous étiez ${ }^{26}$.

On comprend que Madame de Sévigné voudrait savoir si $\mathrm{M}^{\mathrm{me}}$ de Grignan continue à pouvoir prendre la liberté - et/ou en avoir le désir - d'appeler son beau-frère du sobriquet familier de "Seigneur Corbeau ». L'expression présente toutes les caractéristiques de l'estrangement, voire de la ridiculisation carnavalesque, et pourrait donner lieu à une devinette. Mais cette familiarité est ici le signe d'une intimité, d'une bonne entente qui s'exprime en prenant le contrepied de la dignité pour placer cette dernière dans une sorte de distance burlesque, laquelle, paradoxalement, atteste du rapprochement des

24 Dans un article portant sur les effets de réel du langage, et où il se penche sur le procédé d'estrangement, Jakobson note que la fonction réaliste de la défamiliarisation consiste soit à importer du registre familier dans le registre non familier, soit à importer du registre neutre ou soutenu dans le registre familier : "Les tropes nous rendent l'objet plus sensible et nous aident à le voir. En d'autres termes, lorsque nous cherchons le mot juste qui pourrait nous faire voir l'objet, nous choisissons un mot qui nous est inhabituel, au moins dans ce contexte, un mot violé. Ce mot inattendu peut être aussi bien l'appellation figurative que l'appellation propre : il faut savoir laquelle des deux est en usage. Nous avons mille exemples, surtout dans l'histoire du vocabulaire obscène. Appeler l'acte par son propre nom, c'est mordant, mais dans un milieu habitué aux mots grossiers, le trope, l'euphémisme agira d'une manière plus forte et plus convaincante ». (Roman Jakobson, " Du réalisme artistique ", in Théorie de la littérature. Textes des formalistes russes, op. cit., p. 101)

25 Cf. mes articles : "Introduction ", Les Emotions publiques et leur langage au XVII siècle, Hélène Merlin-Kajman (éd.), Littératures classiques, n 68, hiver 2009 ; « Le partage du sensible dans les lettres de Madame de Sévigné », lors de la journée d'étude "Connivences épistolaires ? autour de Madame de Sévigné (lettres de l'année 1671) ", Université Lyon 3, $1^{\text {er }}$ décembre 2012, publication en ligne : http://facdeslettres.univ-lyon3.fr/recherche/gadges/publications/ le-partage-du-sensible-dans-les-lettres-de-l-annee-1671-de-la-correspondance-de-mme-desevigne-625308.kjsp?RH=1206110864985.

26 Madame de Sévigné, lettre du 9 février 1671, in Correspondance, t. I, mars 1646-juillet 1675, Roger Duchêne (éd.), Paris, Gallimard, «Bibliothèque de la Pléiade », 1972, p. 154. 
deux protagonistes de l'échange familier : rapprochement, parce qu'ils sont alors tous deux, dans une égalité rieuse, des mortels. L'expression familière cite la dignité ("Seigneur ") et la ravale au-dessous de l'humain ("Corbeau ») ; et la superposition du « au-dessus » de la dignité et du « au-dessous » de l'animal produit ici ce qu'on pourrait appeler un effet de commune mortalité - à la façon des pensées de Marc Aurèle, quoique plus chaleureux car lié à un sentiment partagé et joueur ${ }^{27}$ !

Bien sûr, il s'agit là d'une sorte de degré zéro de l'estrangement. Il réapparaît souvent dans la correspondance de $\mathrm{M}^{\mathrm{me}}$ de Sévigné, et de façon plus sophistiquée, articulé à une perspective morale et un regard sarcastique sur le monde social. Dès qu'il y a naissance et mort notamment, quelque chose des apparences sociales se lézarde et laisse transparaitre l'étrangeté d'un memento mori souvent burlesque.

C'est que, même dans l'Europe chrétienne, la dignité n'est pas seulement sociale mais pour ainsi dire anthropologique - ou méta-sociale, si l'on préfère -, car elle engage les conditions d'existence, ou de possibilité, de la société ellemême. Toute société humaine doit, pour organiser sa survie, instituer sa permanence au moyen de la culture, du symbolique ${ }^{28}$, c'est-à-dire combattre la loi biologique de la mortalité humaine. Cette nécessité culturelle provient de l'absence d'un programme génétique qui, comme pour les autres animaux, assurerait seul la survie sociale : ce niveau de nécessité est généralement oublié par l'histoire sociale. L'être humain semble avoir toujours donné lieu à une perception dédoublée : l'une qui reconnaît dans sa forme un signe d'identité commune et en quelque sorte digne du nom d'homme; l'autre qui voit en lui - ou pratique sur lui - une autre égalité, mais dans l'informe en quelque sorte, l'informe du corps que Bakhtine appelle grotesque. Hiérarchies et distances respectueuses d'un côté, proximité familière ou outrages de l'autre se greffent sur ces deux versants. Les sociétés ont toujours inventé des signes de dégradation faisant ressortir l'informe : et ces signes oscillent de la proximité amicale ou amoureuse à l'hostilité injurieuse et « estrangeante ». Dépouillée de son caractère utopique, la familiarité décrite par Bakhtine nous permet ainsi d'apercevoir un champ immense de l'expérience sociale, champ d'une familiarité de l'estrangement si je puis dire, qui oscille donc entre une couleur heureuse et une couleur au contraire sinistre, malgré le rire qui l'accompagne souvent.

Or, il est une autre région des situations humaines où l'estrangement prend une couleur franchement tragique. Nous pouvons l'aborder par un autre passage de Carlo Ginzburg. Une citation de Proust évoquant « le côté Dostoïevski des

27 Il faudrait plutôt rapprocher ce sentiment de ce qu'Auerbach a appelé le " créaturel ».

28 Cf. Maurice Godelier, Au fondement des sociétés humaines. Ce que nous apprend l'anthropologie, Paris, Albin Michel, 2007. 
Lettres de $\mathrm{M}^{\mathrm{me}}$ de Sévigné ${ }^{29}$ » amène en effet l'historien à distinguer la forme d'estrangement pratiquée par l'auteur de $L a$ Recherche et celle qu'il vient d'étudier à partir de Marc Aurèle et Guevara. Si cette dernière, rappelle-t-il, est un " moyen de dépasser les apparences et d'atteindre une compréhension plus profonde de la réalité ${ }^{30}$ ", " [l]'objectif de Proust est en un certain sens celui, opposé, de protéger la fraîcheur des apparences contre l'intrusion des idées, en présentant les choses dans l'ordre de nos "perceptions", non encore contaminées par des explications causales ${ }^{31}$ ». Carlo Ginzburg confronte alors l'estrangement selon Proust et l'estrangement selon Chklovski, mais cette fois-ci, concernant ces deux contemporains, le rapprochement est contextuel. L'historien les réinscrit en effet dans le contexte historique particulier du développement de la vie urbaine moderne. Si, d'un côté, ce développement s'est " accompagn[é] d'une énorme intensification de notre vie sensorielle ${ }^{32}$ ", il ne faut pas ignorer qu'il va également de pair avec l'" appauvrissement qualitatif de notre expérience ${ }^{33}$ ", automatisation de la perception pour Chklovski qui voulait donc la combattre par l'estrangement ou " représentations préconstituées " pour Proust qui voulait leur " faire barrage » en leur opposant une sorte d' "immédiateté impressionniste ${ }^{34}$ ". Le concept d'estrangement sort ainsi de son cadre formaliste et intemporel.

C'est ici que je vais introduire ma seconde bifurcation, qui va rejoindre, mais de façon seulement asymptotique, ma bifurcation précédente.

Walter Benjamin a abordé la question de l'appauvrissement de l'expérience dans deux textes différents, "Le Conteur " et "Expérience et pauvreté ». Ce n'est pas à la vie urbaine qu'il la rapporte, mais à la Première Guerre mondiale, pourtant l'" une des expériences les plus effroyables de l'histoire universelle ${ }^{35}$ » :

$[\mathrm{N}]$ 'a-t-on pas constaté que les gens revenaient muets du champ de bataille? Non pas plus riches, mais plus pauvres en expérience communicable. Ce qui s'est répandu dix ans plus tard dans le flot des livres de guerre n'avait rien à voir avec une expérience quelconque, car l'expérience se transmet de bouche à oreille. Il n'y avait à cela rien d'étonnant. Car jamais expériences acquises n'ont été aussi radicalement démenties que l'expérience stratégique par la guerre de

29 Cité par Carlo Ginzburg, À distance, op. cit., p. 31. Ce n'est évidemment pas un hasard que nous retrouvions ici $\mathrm{M}^{\mathrm{me}}$ de Sévigné. Notons aussi que voilà rapprochées ses lettres, remplies de notations discordantes qui singularisent, donc défamiliarisent, les éléments qu'elle y fait rentrer, notamment par la familiarité burlesque, et l'œuvre de Dostoïevski, dans laquelle Bakhtine a reconnu la culture carnavalesque.

30 Ibid., p. 32.

31 Ibid., loc. cit.

32 Ibid., p. 33.

33 Ibid., loc. cit.

34 Ibid., p. 32.

35 Walter Benjamin, "Expérience et pauvreté », in Euvres, vol. 2, trad. Maurice de Gandillac, Rainer Rochlitz et Pierre Rusch, Paris, Gallimard, «Folio essais », 2000, p. 365. 
position, l'expérience économique par l'inflation, l'expérience corporelle par l'épreuve de la faim, l'expérience morale par les manœuvres des gouvernants. Une génération qui était encore allée à l'école en tramway hippomobile se retrouvait à découvert dans un paysage où plus rien n'était reconnaissable, hormis les nuages et, au milieu, dans un champ de forces traversé de tensions et d'explosions destructrices, le minuscule et fragile corps humain ${ }^{36}$.

Les situations vécues pendant la Première Guerre mondiale sont si méconnaissables qu'elles n'entrent dans aucune perception ordinaire; elles produisent un estrangement si radical qu'il détruit toute capacité d'acquérir de l'expérience, c'est-à-dire de reconnaître, dans le vécu, la matière d'une expérience transmissible, communicable dans les formes usuelles de la narration.

La remarque de Benjamin s'alimente évidemment, on l'a souvent relevé, aux recherches parallèlement menées par Freud sur les névroses de guerre et sur le traumatisme, qui ont pour particularité de figer les représentations et de leur conférer un caractère de répétition qui n'est pas sans rapport avec l'automatisme auquel Chklovski oppose le procédé d'estrangement. Et pourtant, comme la brève description de Benjamin nous le fait vivement comprendre, le trauma constitue certainement la plus violente des situations d'estrangement. Nous voyons bien une nouvelle fois l'opposition entre automatisme et estrangement se troubler, et un nouveau chiasme apparaître. D'un côté, l'expérience selon Benjamin est ce qui est à la fois reconnaissable et remarquable, mémorable : du point de vue littéraire, c'est la matière du conteur. De l'autre, la situation traumatique est ce qui pulvérise les perceptions habituelles et introduit aux comportements automatiques, figés, désinvestis affectivement.

Avec le trauma, on entre "dans un domaine où les représentations, les garanties, les idéaux, les légitimités sont réduits à néant ${ }^{37}$ " : dans leur livre Histoire et trauma, les psychanalystes Françoise Davoine et Jean-Marc Gaudillière rappellent que "Jacques Lacan donne à ce domaine, conventionnellement, le nom de "Réel" : ce qui ne connaît ni nom, ni image, et "fait retour toujours à la même place", hors de la symbolisation ». Le Réel, écrivent-ils encore,

fait irruption là où ne fonctionnent plus les oppositions qui structurent notre réalité commune, le dedans et le dehors, l'avant et l'après ; là où sont bafouées les garanties qui fondent le lien social ${ }^{38}$.

Il est frappant que cette citation apparaisse dans un passage de leur livre consacré à Wittgenstein, «le dernier né d'une fratrie de huit enfants ${ }^{39}$ " dont " les trois frères aînés s'étaient suicidés ». Les auteurs inscrivent en effet leur

36 Ibid., loc. cit. Ce passage est repris à la lettre dans «Le Conteur ", in Euvres, vol. 3, éd. cit., p. 115-116.

37 Françoise Davoine et Jean-Max Gaudillière, Histoire et Trauma. La folie des guerres, Paris, Stock, 2006, p. 61.

38 Ibid., loc. cit.

39 Ibid., loc. cit. 
propre démarche analytique dans le sillage des recherches de Wittgenstein. Car lorsqu' on est face au Réel, "[1]e cas particulier constitue ici le seul niveau de pertinence, et n'en déplaise à la loi des grands nombres, nous n'avons pas à en avoir honte" :

"Le monde est tout ce qui fait cas. " Ainsi commence le Tractatus composé par Wittgenstein tandis qu'il combattait dans les rangs de l'armée autrichienne pendant la guerre de 14. Une variation sur la dernière phrase : «Ce qu'on ne peut pas dire, il faut le taire ", tout aussi célèbre, a inspiré l'exergue du présent livre : "Ce dont on ne peut pas parler, on ne peut pas le taire ${ }^{40}$."

Or, il est non moins frappant de constater à quel point la réflexion de Wittgenstein passe constamment par la figure de l'estrangement. Je cite, un peu au hasard de mon propre plaisir :

"Un enfant nouveau-né n’a pas de dents ", - «Une oie n’a pas de dents " - "Une rose n'a pas de dents ", - Cette dernière constatation - aimerait-on dire - est manifestement vraie! Elle est même plus sûre que celle qui énonce qu'une oie n'en a pas. Et pourtant ce n'est pas aussi clair. Car où pourraient se trouver les dents de la rose ? L'oie n’en a pas dans sa mâchoire. Et, bien entendu, elle n'en a pas non plus dans ses ailes; mais personne ne prétend cela, quand on dit qu'elle n'a pas de dents. Mais supposons qu'on dise : la vache mâche sa pâture et alors de sa bouse fume la rose, donc la rose a des dents dans la gueule d'une bête. Ceci ne serait point absurde, parce qu'on ne sait pas du tout, de prime abord, où chercher des dents dans une rose ${ }^{41}$.

La différence entre ce passage et les pensées de Marc Aurèle saute aux yeux. La valeur heuristique de l'estrangement, ici, ne procède d'aucun mouvement de dégradation, de rabaissement. Wittgenstein s'affronte, avec les moyens de la logique et de la philosophie du langage, non pas tant à la réalité qu'au Réel, à la terreur de l'étrange, c'est-à-dire, selon Lacan, à l'Impossible :

Nous apprenons le mot " rouge " dans des circonstances bien déterminées. Certains objets sont habituellement rouges et conservent leurs couleurs; la plupart des gens s'accordent avec nous pour juger des couleurs. Supposez que tout cela change : inexplicablement je vois le sang, parfois d'une certaine couleur, parfois d'une autre, et les gens qui m'entourent portent des jugements différents [...] L'atmosphère qui enveloppe ce problème est terrifiante. D'épais brouillards de langue entourent le point problématique. Il est à peu près impossible de se frayer un passage jusqu'à lui ${ }^{42}$.

On le comprend, les deux psychanalystes établissent ainsi une relation à la fois métonymique et métaphorique entre le marquage traumatique de la vie de Wittgenstein, son goût pour le cas singulier et sa mise à l'épreuve du langage

40 Ibid., p. 59.

41 Ludwig Wittgenstein, Investigations philosophiques, in Tractatus logico-philosophicus, Paris, Gallimard, « Tel », 1997, p. 354.

42 Ludwig Wittgenstein, Notes sur l'expérience privée et les "sense data ", Mauvezin, Trans-EuropRepress, 1989, p. 36. 
ordinaire par des formulations qui le font passer insensiblement d'un régime familier à un régime " estrangé ». Ainsi se trouve encore justifié l'importance du cas particulier et des détails symptomatiques, ces " petits riens » qui « enrayent les machines signifiantes [et] détraquent l'esprit de sérieux ${ }^{43}$ ». Ce qu’ils suggèrent, c'est que Wittgenstein, en somme, fait ressortir les traces traumatiques que le langage peut abriter autant qu'il recherche ses ressources thérapeutiques ${ }^{44}$.

Nous voici donc, avec cette question du Réel et du trauma, face à une autre piste pour comprendre la fonction possible du procédé littéraire de l'estrangement : fonction d'exploration analogique et d'élaboration réparatrice. Or, la piste nous ramène à Carlo Ginzburg. Car, évoquant le caractère « [a] chronique " et " atopique " du symptôme traumatique, Françoise Davoine et Jean-Max Gaudillière font surgir une nouvelle référence :

[V]oilà que débarque par son truchement un bout d'histoire échappé à l'Histoire, au croisement du singulier et du pluriel, sous la forme d'un "social en train de se faire ", nous dit l'historien Jacques Revel à propos des travaux des historiens de la micro-histoire, comme Giovanni Levi ou Carlo Ginzburg ${ }^{45}$.

Ici, Françoise Davoine et Jean-Max Gaudillière rapprochent leur travail d'analyste de celui des historiens de la microstoria. Le rapprochement peut paraître surprenant : quel rapport peut-il y avoir entre l'histoire, qui vise la connaissance du passé, et la psychanalyse, qui, engagée avec un patient, a un but thérapeutique? La réponse est à chercher dans l'historicité particulière du trauma, historicité qui requiert, selon les auteurs, une écoute, une réception également spécifiques :

Toute interruption dans la transmission qui relie entre eux les hommes cherche paradoxalement les voies d'une inscription. Le plus souvent, cette dynamique semble se heurter à l'irréparable. Mais la folie constitue une des relations sociales vouées à ce travail, dur et précis, au contact de l'impossible. Elle met en mouvement une co-recherche, dont l'éventuel analyste occupe la seconde place. Il fait donc partie du champ à analyser ${ }^{46}$.

Il me semble que la position ici décrite rend admirablement compte de tout un versant du travail de Carlo Ginzburg, à commencer par l'importance que revêt pour lui la chaîne des noms propres qui jalonnent les pistes

43 Françoise Davoine et Jean-Max Gaudillière, op. cit., p. 59.

44 " [L]e philosophe, au prix de sévères crises suicidaires, passe de la conclusion du Tractatus à l'élaboration d'une philosophie qui vaut pour lui "comme une thérapie" où, même "quand l'outil du nom N. est cassé", il est toujours possible de montrer les morceaux, et d'imaginer "une convention qui donne un lieu au nom cassé, dans le jeu de langage, même quand l'outil n'existe plus, même quand le nom n'a plus de sens, même quand son porteur a cessé d'exister." " (Ibid., p. 60. La citation de Wittgenstein, retraduite par les auteurs, est tirée des Investigations philosophiques, op. cit., $\$ 41$, p. 135)

45 Françoise Davoine et Jean-Max Gaudillière, op. cit., p. 57.

46 Ibid., p. 57-58. 
"tortueuses " suivies par l'historien, chacun d'eux faisant l'objet non seulement d'un examen attentif mais aussi d'une sorte de souci attentionné, comme s'il s'agissait non seulement de connaissance, mais aussi de mémoire et d'inscription symbolique ${ }^{47}$. C'est ainsi que le chapitre "Sur les traces d'Israël Bertuccio » du Fil et les traces trouve son point de départ dans ce nom propre prononcé par Julien Sorel, et de là, remonte à un personnage historique réel, Bertuccio Isarello, probable chef d'une conspiration vénitienne dont le nom a été modifié par sa circulation dans les textes et dont la " voix, étouffée sur le gibet, n'a pu nous parvenir ${ }^{48} »$. En termes de connaissance historique, le gain n'est guère évident : mais de déchirure en déchirure, dans le sillage d'un nom étranger et estropié, puis d'un destin tragique, quelque chose comme un tort a été réparé, de la transmission s'est rétablie. C'est un détail du Rouge et le Noir, un nom entendu dans une tragédie de Casimir Delavigne et remémoré par Julien, donc, qui sert de point de départ : pur signe littéraire qui renvoie lui-même à une tragédie de Byron. Mais il fonctionne comme le symptôme traumatique : il encapsule un morceau d'histoire oublié, un fragment de Réel, enfin recueilli et accueilli par l'historien-analyste.

Carlo Ginzburg a souvent fait référence au concept élaboré par Chklovski. Dans un autre chapitre du Fil et les traces, "Détails, gros plan, micro-analyse ", il qualifie ainsi d'estrangé, " estrangé et pour ainsi dire mécanique ${ }^{49}$ ", le regard, " que Proust compare à l'objectif impassible de l'appareil photographique $^{50}$ ", du narrateur apercevant soudain et pour un bref instant, dans Le Côté de Guermantes, "sur le canapé, sous la lampe, rouge, lourde et vulgaire, malade, rêvassant, promenant au-dessus d'un livre des yeux un peu fous, une vieille femme accablée que je ne connaissais $\mathrm{pas}^{51} »$ : sa grand-mère. Le texte communique ainsi le choc produit par la superposition de la méconnaissance et de la reconnaissance d'une personne aimée, l'étrangeté causée par sa vision pourtant en principe familière : l'estrangement traduit cette discordance violente. "Rouge, lourde et vulgaire..." : nous voici en outre à la limite de la dégradation outrageante qui accompagne, nous l'avons vu, l'estrangement chez Marc Aurèle ou dans la culture carnavalesque. Mais dans l'exemple

47 Cf. Martin Rueff, "L'historien et les noms propres ", Critique, "Sur les traces de Carlo Ginzburg ", Patrizia Lombardo et Martin Rueff (éd.), nº 769-770, juin-juillet 2011, p. 529-531.

48 Carlo Ginzburg, "Sur les traces d'Israël Bertuccio ", in Le fil et les traces. Vrai faux fictif, Paris, Verdier, 2010, p. 248.

49 Carlo Ginzburg, "Détails, gros plan, micro-analyse », in ibid., p. 341.

50 Ibid., loc. cit. Voici la citation de Proust : "Ce qui, mécaniquement, se fit à ce moment dans mes yeux quand j'aperçus ma grand-mère, ce fut bien une photographie. " (Cité par Carlo Ginzburg, ibid., loc. cit.)

51 Cité par Carlo Ginzburg, ibid., loc. cit. Les citations proviennent de : Marcel Proust, À la Recherche du temps perdu, t. II, Le Côté de Guermantes, Pierre Clarac et André Ferré (éd.), Paris, Gallimard, "Bibliothèque de la Pléiade », 1954, p. 140-141. 
proustien, l'effraction traumatique est sensible, car le lecteur sent ou sait que le narrateur est ou sera affecté : malgré la métaphore de la photographie qui veut signifier un enregistrement objectif de l'image, cette objectivation s'interprète assez facilement comme la neutralisation momentanée d'un excès traumatique ; et la description laisse du reste filtrer, au-delà des ravages physiques de la maladie, ses ravages psychiques sur la vieille femme, donc aussi sur le narrateur dont le lecteur sait bien qu'il est à la fois celui qui a vu et celui qui raconte, c'est-à-dire celui pour lequel le temps a réveillé l'affect, la peine que l'impact de la vision traumatique avait au contraire inhibés.

Mais l'exemple le plus fructueux du travail du « Réel » dans la pensée de Carlo Ginzburg est sans doute celui d'un chapitre de Rapports de force, «Déchiffrer un espace blanc ${ }^{52}$ ». Carlo Ginzburg rappelle le jugement de Proust sur le blanc typographique " probablement le plus célèbre dans l'histoire du roman ", celui qui « se trouve dans L'Éducation sentimentale de Flaubert entre les chapitres V et VI de la troisième et dernière partie de l'œuvre ", et qui sépare le moment où Frédéric reconnaît, dans l'agent de police qui vient de tuer l'insurgé Dussardier, son ancienne connaissance Sénécal ("Et Frédéric, béant, reconnut Sénécal ») et une toute autre séquence narrative : «Il voyagea ${ }^{53}$ ». Pour Proust, Flaubert réussit à "met[tre] en musique » la dimension historique qui, chez Balzac, reste parasitée par le réalisme documentaire. Carlo Ginzburg conteste cette perspective en opérant une lecture référentielle de cet espace blanc - en le documentant, en somme, et ceci, deux fois. D'une part, il rapproche le procédé littéraire non pas de la musique - qui, chez Proust, constitue une métaphore intemporelle de l'art de Flaubert - mais de la technique du montage cinématographique et de la photographie (comme dans l'exemple de la grand-mère de Proust) alors en plein développement (pour la seconde) ou en pleine genèse (pour le premier) : il y aurait donc là une identité de procédé explicable historiquement entre la littérature et les arts modernes de l'image. D'autre part, Carlo Ginzburg se rallie au jugement de Maurice Agulhon pour qui cette ellipse narrative fait remarquablement « sentir la coupure que toute une partie de la société française a ressentie lorsque la dictature bonapartiste a remplacé la République ${ }^{54} "$ : l'espace blanc n'est pas une musique, mais une transcription littéraire adéquate d'un contexte événementiel bien déterminé.

Mais un blanc typographique, une ellipse narrative, peuvent-ils vraiment être documentés ? Peuvent-ils transcrire des événements ou des mutations historiques ? Est-il certain que cet exemple illustre « la richesse cognitive de

52 Carlo Ginzburg, Rapports de force. Histoire, rhétorique, preuve, Paris, Gallimard/Seuil, « Hautes Études", 2003.

53 Carlo Ginzburg, "Déchiffrer un espace blanc », in ibid., p. 87.

54 Ibid., p. 95. 
l'œuvre de Flaubert ${ }^{55}$ " ? Ne faut-il pas plutôt penser, comme Proust, qu'il s'agit davantage de "musique " que de réalité historique ${ }^{56}$ ? Ou pour être plus précis, ne faut-il pas comprendre qu'ici, l'écrivain a trouvé une modalité d'inscription juste du trauma, entre le symptôme aporétique dont souffre le " fou » et sa reprise ou sa relance dans l'écoute et la parole du psychanalyste (ou de l'historien) ? Un blanc, rien qu'un blanc, c'est-à-dire une façon d'adresser esthétiquement la trace traumatique à ses lecteurs - de la présenter, non de représenter le passé. "Achronique ", " atopique " : ainsi s'expliquerait la capacité de la littérature à transiter dans l'histoire...

L'un des derniers textes cités par Carlo Ginzburg dans "Déchiffrer un espace blanc " est, significativement, un texte de Marc Bloch :

Il est probable que tant que je vivrai, à moins que je ne finisse mes jours dans l'imbécillité, je n'oublierai jamais le 10 septembre 1914. Mes souvenirs de cette journée ne sont pourtant pas extrêmement précis. Surtout ils s'enchaînent mal. Ils forment une série discontinue d'images, à la vérité très vives, mais médiocrement coordonnées, comme un rouleau cinématographique qui présenterait par places de grandes déchirures et dont on pourrait, sans que l'on s'en aperçût, intervertir certains tableaux ${ }^{57}$.

Plus qu'une métaphore de la connaissance, les variations sur la métaphore de la photographie ou du déroulement cinématographique ont peut-être constitué, dans le contexte du $\mathrm{XX}^{\mathrm{e}}$ siècle, des figures adéquates pour transmettre un choc traumatique, entre automatisme aveugle et estrangement radical.

«Pourquoi [les historiens] devraient-ils perdre leur temps avec l'estrangement ou tout autre de ces concepts élaborés par les théoriciens de la littérature $^{58}$ " ? se demande Carlo Ginzburg en conclusion de son parcours portant sur l'estrangement. Il donne alors cette réponse qui combat le positivisme autant que le scepticisme de la modernité :

L'estrangement me semble susceptible de constituer un antidote efficace à un risque qui nous guette tous : celui de tenir la réalité (nous compris) pour sûre. Les implications antipositivistes d'une telle observation sont évidentes. Mais en soulignant les enjeux cognitifs de l'estrangement, je voudrais m'opposer aussi avec la plus grande clarté à certaines théories en vogue, qui tendent à brouiller jusqu'à les rendre indistinctes les frontières entre l'histoire et la fiction ${ }^{59}$.

À mes yeux, le point par lequel Carlo Ginzburg se sépare le plus totalement et le plus effectivement du scepticisme lorsqu'il se penche sur les textes et les procédés littéraires n'est peut-être pas la confiance continûment accordée

55 Ibid., p. 97. Je souligne.

56 Cité par Carlo Ginzburg, ibid., p. 87.

57 Marc Bloch, "La bataille de la Marne ", Annales. Économies, Sociétés, Civilisations, $22^{\mathrm{e}}$ année, $\mathrm{n}^{\circ} 3,1967$, p. 474.

58 Carlo Ginzburg, À Distance, op. cit., p. 34-35.

59 Ibid., p. 36. 
dans la valeur « cognitive " de ses enquêtes. C'est plutôt le fait que son travail opère en fait toujours à la jonction de la réalité et du Réel en procédant à leur mise en relation. Pour le dire autrement, l'historien se double toujours d'un témoin. Or, qu'est-ce qu'un témoin, sinon celui qui a vu les faits, qui en a été proche et peut les re-présenter ? Selon une formule du Fil et les traces, "[1]e regard rapproché nous permet de saisir quelque chose qui échappe à la vision d'ensemble et réciproquement ${ }^{60} "$ : le rapprochement est le pendant nécessaire de l'estrangement, sans quoi l'estrangement reste plus défensif que cognitif et laisse s'échapper la part d'ombre qui enveloppe la réalité quand elle se trouve avoir été elle-même estrangée - part d'ombre et zone détruite auxquelles j’ai ici donné le nom de Réel dans le sillage de la psychanalyse. Dans "Sorcières et chamans ", Carlo Ginzburg a raconté comment il avait découvert l'interrogatoire d'un benandante: "Les choses se sont passées comme si tout d'un coup j'avais reconnu un document qui m'était parfaitement inconnu l'instant auparavant ${ }^{61}$ ». Quelques détails étranges mais symptomatiques, tout à fait anormaux par rapport aux autres procès d'Inquisition et à la culture des juges, se fraient un chemin dans les aveux qui sortent des bouches des accusés. L'historien qui entend le signe adressé à travers ces textes fait le pari que malgré les corps torturés, une expérience presque détruite et inaudible peut être captée, saisie. À partir de là, le travail consiste non seulement à restituer la vérité référentielle (des rites, des mythes chamaniques), mais aussi à réinsérer dans la trame symbolique ce qui, sans l'historien, ne pouvait plus s'y inscrire.

Quelques pages plus haut, Carlo Ginzburg avait rassemblé les éléments autobiographiques qui permettaient peut-être d'expliquer ce premier objet de recherche. L'un d'eux lui a été signalé un jour par un ami qui lui «a fait remarquer que le choix d'étudier la sorcellerie, et en particulier les victimes de la persécution de la sorcellerie, n'avait rien d'étonnant de la part d'un Juif qui avait connu la persécution » :

Cette observation toute simple me laissa interdit. Comment avais-je pu laisser échapper un fait si évident ? [...] Aujourd'hui je suis enclin à voir dans tout cela l'effet du refoulement. Comme Freud nous l'a appris, ce qui est à la fois évident et caché, c'est ce que l'on ne veut pas voir ${ }^{62}$.

Il est frappant de constater que cette dimension d'évidence et de secret correspond assez bien au mouvement de reconnaissance de quelque chose d'inconnu décrit par l'historien face aux détails inouïs de l'interrogatoire du benandante. Le désir de connaître est bordé par cette double puissance de méconnaissance et de reconnaissance et s'accompagne alors du désir de faire rentrer dans l'histoire humaine - dans l'histoire racontée, dans l'histoire

60 Carlo Ginzburg, Le fil et les traces, op. cit., p. 389.

61 Carlo Ginzburg, "Sorcières et chamans ", in ibid., p. 436.

62 Ibid., p. 431-432. 
mémorable - les êtres humains qui avaient été expulsés de l'histoire agie, qu'elle avait défigurés, frappés d'étrangeté. On peut faire l'hypothèse que l'historien communique alors avec eux par sa propre "étrangeté " et son propre " réel " de la même manière que le psychanalyste communique avec son patient par son propre point de trauma. Et pour reprendre la perspective de Françoise Davoine et Jean-Max Gaudillière, ici, les enchaînements causaux ordinaires ne suffisent pas. Il faut parcourir l'histoire en tous sens en affrontant ses zones d'inquiétante étrangeté et d'anomie familière.

Pour conclure, je voudrais souligner que comme Chklovski, Carlo Ginzburg et tant d'autres, je suis évidemment favorable au procédé littéraire de l'estrangement ${ }^{63}$. Mais en introduisant deux bifurcations dans le trajet de l'historien, j'ai voulu suggérer d'en distinguer tendanciellement deux réalisations. Dans un cas, le mouvement d'estrangement consiste à dégrader, défigurer, voire expulser : la finitude de la mort reconnaissable derrières les formes stabilisées, familières, de la culture, autorise l'outrage qui exile tel ou tel être humain, telle ou telle situation sociale, hors d'une commune humanité. Dans l'autre cas, il s'agit au contraire, par l'estrangement, de rejoindre le point de Réel, d'étrangeté radicale, qui n'arrête pas de strier l'histoire, individuelle comme collective. C'est cette démarche de rapprochement que, pour ma part, je reconnais dans la démarche d'estrangement de Carlo Ginzburg.

\author{
Hélène Merlin-Kajman \\ EA 174 \\ Université de la Sorbonne Nouvelle-Paris 3 \\ Helene.Merlin-Kajman@univ-paris3.fr
}

\begin{abstract}
Résumé
Carlo Ginzburg n'a pas seulement valorisé le procédé d'estrangement, il a aussi valorisé le regard rapproché. Nous suivons ici les arrière-plans théoriques et pratiques des deux démarches en observant, d'une part, comment l'estrangement peut jouxter la familiarité carnavalesque voire outrageante qui dégrade ce dont elle s'empare, aboutissant à l'exclusion violente de certains sujets de l'histoire humaine ; d'autre part, comment le regard rapproché, pour l'historien comme pour l'écrivain, peut faire entrer en contact avec l'étrangeté radicale du choc traumatique, (le « Réel » au sens lacanien du terme), c'est-à-dire lui frayer un passage et, de la sorte, réinscrire les zones d'ombre dans la trame symbolique de l'histoire, c'est-à-dire aussi renouer le fil de la transmission.
\end{abstract}

63 J'ai du reste écrit moi-même un roman qui le mobilisait : La Désobéissance de Pyrame, Paris, Belin, 2009. Cf. aussi mon article : "La fiction "classique" : le plaisir du dépaysement et de l'interrogation morale (La désobéissance de Pyrame) ", Papers on French Seventeenth Century Literature, "Les représentations du XVII siècle dans la littérature pour la jeunesse contemporaine : patrimoine, symbolique, imaginaire " (actes du colloque de Lyon, 12-13 mai 2011), Marie Pérouse-Battelo et Edwige Keller-Rahbé (éd.), vol. XXXIX, nº 77, 2012. 


\section{Mots-clés}

Familiarité, réalité/Réel, trauma, expérience, fiction, transmission.

\section{Abstract}

Carlo Ginzburg dit not only valorise the process of "estrangement" but also the close insight. Here we follow the theoretical and practical backgrounds of these two notions, observing, first, how the "estrangement" is very close to the familiarity described by Bakhtin as "canavalesque", that is to say a familiarity which may expulse as stranger what (and whom) it has insulted; then, at the contrary, how the close insight may procure to the historian as to the writer an approach to what Lacan calls the Real, the trauma: and in this way, make room for its reinscription in the symbolic framework of history, rather than its traumatical renewal.

\section{Keywords}

Familiarity, reality/the Real, trauma, experience, fiction, transmission. 